\title{
TRIDIRECTIONAL TRANSMISSION SYSTEMS USING POLARIZATION CONTROL TECHNIQUE
}

\author{
Shien-Kuei Liaw, Keang-Po Ho*, Fan-Yi Chiang, Fu-Chun Hung \\ Department of Electronic Engineering, \\ National Taiwan University of Science and Technology, \\ No. 43, Sec. 4, Keelung Rd., Taipei, 106, Taiwan, R.O.C.
}

Phone: 886-227376384, Fax: 886227376424,E-mail: skliaw@et.ntust.edu.tw

*StrataLight Communications, Campbell, CA 95008, U.S.A

Summary- Wavelength-division-multiplexed (WDM) based optical networks are indispensable for current and future high-capacity networking infrastructure. As the growth on the demand of information capacity, tri-directional transmission will be another useful solution for network design, signals interchange and cross connect purpose. Conventional systems use different wavelengths for counter-propagating WDM signals to avoid overlapping the spectra. Though the Rayleigh backscattering (RB) can be reduced, it will restrict the maximum spectral efficiency.

Fig. 1 (a) shows the proposed triangle box as a tri-directional transmission module (TTM). It at least consists of three pieces of OCs. Fig. 1 (b) shows the TTM in a tri-directional system with $\lambda_{a}$ travels from point a to point $b, \lambda_{b}$ travels from point $b$ to point $c$ and $\lambda_{c}$ travels from point $c$ to point a. Fig. 2 depicts the experimental setup to study a polarization-controlled wavelength-reused tri-directional transmission. Except one dummy port, the other three signals are imitated as three partly counter-propagating signals with $0 \mathrm{dBm}$ power level at points $\mathrm{a}, \mathrm{b}$ and $\mathrm{c}$. Note that $\lambda_{\mathrm{a}}, \lambda_{b}$, and $\lambda_{c}$ are the same wavelength of equal power. Figure 3 simulates the possible impact of RB signals $\lambda_{b}$ and $\lambda_{c}$ induces homodyne crosstalk to $\lambda_{\mathrm{a}}$ under random polarization. As $\lambda_{\mathrm{a}}$ travels from TMM to point $\mathrm{b}$ (i.e., in SMF2), the crosstalk level of RB signal $\left(\lambda_{b}\right)$ to $\lambda_{a}$ is as large as $-20 \mathrm{~dB}$ (i.e., -50 to $-20 \mathrm{~dB}$ ). Such crosstalk level may induce $1.5 \mathrm{~dB}$ power penalty to $\lambda_{\mathrm{a}}$. Fig. 4 shows the measured BER of $\lambda_{\mathrm{a}}$ as a function of received optical power after pass through SMF1 and SMF2. Compared to back-to-back measurement, the power penalty is $0.4,2.0$, and $4.0 \mathrm{~dB}$ for the best BER with PCs, random polarized without PCs (or shorten as normal PC), and the worst BER with PCs, respectively.

In a tri-directional wavelength-reused system of $50 \mathrm{~km}$ SMF runs $10.0 \mathrm{~Gb} / \mathrm{s}$ bit rate, launching counter-propagating signals with orthogonal polarization using polarization controllers can reduce the impact of Rayleigh backscattering. The results show that power penalty is greatly reduced. The proposed technique may find vast applications in WDM ring networks and tri-directional system where signals add-drop, interchange, as well as cross-connect functions are necessary.
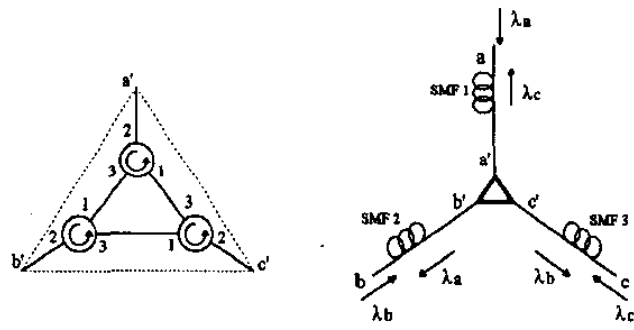

Fig. I (a) The proposed tri-directional transmission module (TTM), (b) TTM in a tri-directional transmission system.

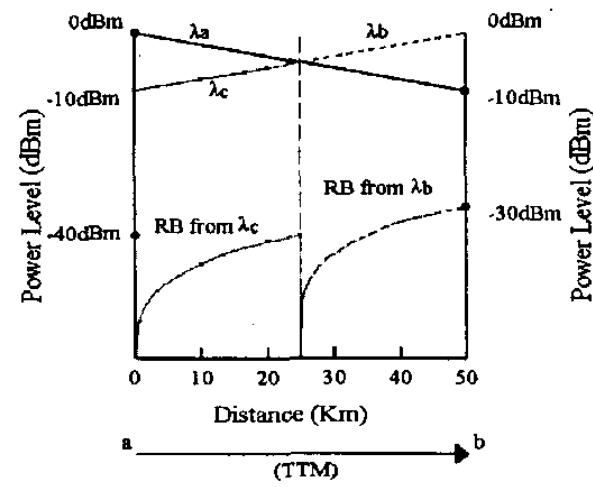

Fig. 3 Simulation result of RB signals $\lambda_{c}$ induces homodyne crosstalk to $\lambda_{a}$ in SMF1; and the RB signals $\lambda_{b}$ induces homodyne crosstalk to $\lambda_{\mathrm{a}}$ in SMF2 .

0-7803-7766-4/03/\$17.00 (C)2003 IEEE

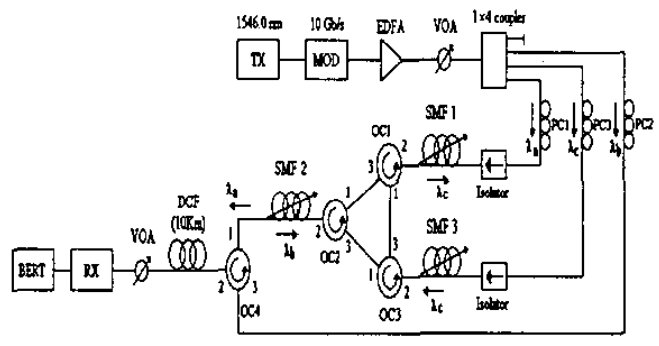

Fig. 2 Experimental setup.

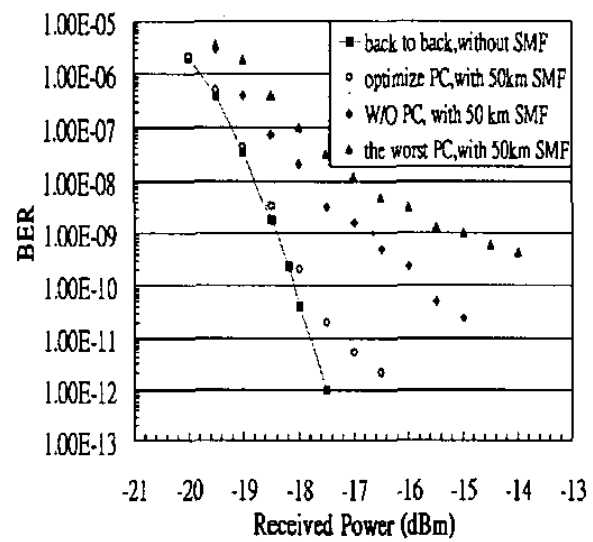

Fig. 4 The measured BER results as a function of received optical power after $50(25+25) \mathrm{km}$ SMF with or without the polarization controllers. 